\title{
The energy scale of cosmic rays detected with LOFAR
}

K. Mulrey ${ }^{1 *}$, A. Bonardi ${ }^{2}$, S. Buitink ${ }^{1,2}$, A. Corstanje ${ }^{1,2}$, H. Falcke ${ }^{2,3,4}$, B. M. Hare ${ }^{5}$, J. R. Hörandel ${ }^{1,2,3}$, T. Huege ${ }^{1,7}$, G. Krampah ${ }^{1}$, P. Mitra ${ }^{1}$, A. Nelles ${ }^{8,9}$, H. Pandya ${ }^{1}$, J. P. Rachen ${ }^{1,2}$, L. Rossetto ${ }^{2}$, O. Scholten ${ }^{5,6}$, S. ter Veen ${ }^{2,4}$, T. N. G. Trinh ${ }^{5,10}$, T. Winchen ${ }^{1}$

${ }^{1}$ Astrophysical Institute, Vrije Universiteit Brussel, Pleinlaan 2, 1050 Brussels, Belgium,

${ }^{2}$ Department of Astrophysics/IMAPP, Radboud University, P.O. Box 9010, 6500 GL Nijmegen, The Netherlands,

${ }^{3}$ NIKHEF, Science Park Amsterdam, 1098 XG Amsterdam, The Netherlands,

${ }^{4}$ Netherlands Institute of Radio Astronomy (ASTRON), Postbus 2, 7990 AA Dwingeloo, The Netherlands,

${ }^{5}$ KVI-CART, University Groningen, P.O. Box 72, 9700 AB Groningen,

${ }^{6}$ Interuniversity Institute for High-Energy, Vrije Universiteit Brussel, Pleinlaan 2, 1050

Brussels, Belgium,

${ }^{7}$ Institut für Kernphysik, Karlsruhe Institute of Technology(KIT), P.O. Box 3640, 76021, Karlsruhe, Germany

${ }^{8}$ DESY, Platanenallee 6, 15738 Zeuthen, Germany

${ }^{9}$ ECAP, Friedrich-Alexander-University Erlangen-Nürnberg, 91058 Erlangen, Germany

${ }^{10}$ Department of Physics, School of Education, Can Tho UniversityCampus II, 3/2 Street, Ninh Kieu District, Can Tho City, Vietnam

E-mail: kmulrey@vub.ac . be

The LOw-Frequency ARray (LOFAR) measures radio emission from extensive air showers. Precise knowledge of the electric field at each antenna in the $30-80 \mathrm{MHz}$ range is obtained using a newly developed, frequency-dependent calibration built on knowledge of the Galactic emission and a detailed model of the signal chain. The energy fluence for each event is then determined, allowing for the calculation of the radiation energy of the air shower. The radiation energy, corrected for geometrical effects, scales quadratically with the energy contained in the electromagnetic component of the air shower. These measurements, combined with predictions that rely only on first-principle electrodynamics, provide an energy estimate for the primary particle. In this contribution we present the radio-based energy scale of cosmic rays detected with LOFAR, and compare it to particle-based energy measurements made using the scintillator array located at the LOFAR core.

36th International Cosmic Ray Conference -ICRC2019-

July 24th - August 1st, 2019

Madison, WI, U.S.A.

${ }^{*}$ Speaker. 


\section{Introduction}

When cosmic rays enter the Earth's atmosphere they interact, generating a cascade of secondary particles which emit coherently in the radio regime. This emission is generated through the charge-excess effect and the transverse current induced by the magnetic field of the Earth. The radio emission is produced primarily by the electromagnetic components of the shower, and is calculated from first principles using classical electrodynamics [1]. With knowledge of the electric field at ground level, the energy fluence and radiation energy of the event can be determined. The radiation energy, once corrected for geometrical effects, scales quadratically with the energy contained in the electromagnetic component of the air shower, thereby providing an energy estimate for the primary cosmic ray. The measured signal is integrated over the whole air shower, and so measurements can be used to perform complete calorimetric energy reconstructions [2, 3].

The LOw Frequency ARray (LOFAR) is well suited to study radio emission from cosmic rays because of its dense antenna spacing. Features of the primary cosmic ray including energy, geometry, and composition are reconstructed with high precision $[4,5,6]$. The frequency range of the low-band antennas (LBAs) used is $30-80 \mathrm{MHz}$. An in situ particle detector array, LORA, is used as a trigger for antenna readout. Until recently, shower parameters like $\mathrm{X}_{\max }$ were reconstructed using radio data, but the overall energy scale was set using particle data measured with LORA. Since a new technique has been developed that provides an absolute, frequency dependent calibration for the LOFAR antennas [7], we move to using radio measurements to set the LOFAR energy scale [8]. In this contribution we present the methods by which the energies of LOFAR air showers are reconstructed, and make a comparison of the energies determined using radio and particle techniques.

\section{Method}

When LORA detects particles from an air shower, 2 ms of LOFAR radio data are saved. The radio data are then cleaned for RFI and calibrated. Finding an absolute calibration for antennas in the range $30-80 \mathrm{MHz}$ has been a challenge due to complications created by electronic noise and uncertainties in antenna characteristics. To address that problem, a new technique was developed using the Galactic emission and a model of the signal chain to provide a frequency-dependent conversion factor from ADC counts to voltage at the antenna feed. Details can be found in [7]. The events used in this analysis have been detected by at least three LOFAR stations, and passed quality cuts on polarization and geometrical reconstruction.

The combination of emission mechanisms in the air showers leads to a complicated radio footprint at ground level. The emission mechanisms are well understood, and the pattern can be reproduced with particle-level, first principle simulations like CORSIKA [9] and the radio plugin CoREAS [10]. In this section two methods of determining cosmic-ray energy based on radio measurements are described. The first is referred to as the direct scaling method, and is what has been used in past LOFAR analyses. The second is a fluence-based method, which follows the approaches described in [2] and [3]. Both methods are based on CoREAS simulations.

\subsection{Direct scaling method}

For each LOFAR event, a set of simulations is generated covering a range of $X_{\max }$ values for 
both proton and iron primaries (Corsika 7.6300 [9], Fluka [11], QGSJetII-04 [12]). The arrival direction used in the simulation is based on timing reconstruction of the event, and the energy used in the simulation is based on the results of a two dimensional LDF fit to the radio data [13]. Each simulation includes a realistic atmosphere, specific to the time and location of the event [14]. Antennas are simulated in a star shape pattern in the antenna plane, and the LOFAR LBA antenna model is applied to the simulated electric field, yielding the voltage at the antenna. The integrated power within a $55 \mathrm{~ns}$ window is calculated. This is interpolated into a two dimensional map. Full details of the method are found in [5]. This map is then fit to LOFAR data using a minimization procedure with free parameters for the core position of the shower and a scale factor for the energy, as

$$
\chi^{2}=\sum_{\text {antennas }}\left(\frac{P_{\mathrm{ant}}-f_{r}^{2} P_{\mathrm{sim}}\left(x_{\mathrm{ant}}-x_{0}, y_{\mathrm{ant}}-y_{0}\right)}{\sigma_{\mathrm{ant}}}\right)^{2}
$$

where $P_{\text {ant }}$ is the measured integrated power, $P_{\text {sim }}$ is the simulated integrated power at $\left(x_{\text {ant }}, y_{\text {ant }}\right)$, $\sigma_{\text {ant }}$ is the noise level in the antenna, $\left(x_{0}, y_{0}\right)$ is the core position, and $f_{r}$ is the energy scaling factor. The simulation with the lowest $\chi^{2}$ value is taken as the "truth." The cosmic-ray energy, $E_{\text {dir.scal., }}$ is determined by multiplying the simulated energy by the scale factor.

\subsection{Fluence-based method}

The fluence-based method uses the best-fit simulation as determined by the direct scaling method. With the CoREAS simulation closest to the true event, we follow the procedure outlined in [2] to determine the radiation energy. The energy fluence at each antenna is calculated using

$$
f(\vec{r})=\varepsilon_{0} c \Delta t \sum_{i} E^{2}\left(\vec{r}, t_{i}\right)
$$

where $c$ is the speed of light in vacuum, $\varepsilon_{0}$ is the vacuum permittivity, and $\Delta t$ is the sampling interval of the time and position dependent electric field $E(\vec{r}, t)$. The radiation energy is then calculated as

$$
E_{\mathrm{rad}}=\int_{0}^{2 \pi} \mathrm{d} \phi \int_{0}^{\infty} \mathrm{d} r r f(r, \phi)
$$

where we make use of the radial symmetry of the geomagnetic and charge excess distributions, and use the fluence where $\phi$ is along the $\vec{v} \times \vec{v} \times \vec{B}$ axis in the shower plane. This results in an overestimate of the radiation energy by $3.36 \%$ [15], which is corrected for. The strength of the radio emission depends on the local magnetic field, which affects the geomagnetic component of the emission. The radiation energy is corrected by

$$
S_{R D}=\frac{E_{\mathrm{rad}}}{\left(a^{\prime 2}+\left(1-a^{\prime 2}\right)\right) \sin ^{2} \alpha\left(\frac{B_{\mathrm{Earth}}}{0.243 \mathrm{G}}\right)^{1.8}}
$$

where $a^{\prime}=a /\left(B_{\text {Earth }} / 0.243 \mathrm{G}\right)^{0.9}, a$ is a parametrization of the charge-excess fraction based on the simulated $\mathrm{X}_{\max }$, and $\alpha$ is the angle between $\vec{v}$ and $\vec{B}$. $B_{\text {Earth }}=0.496 \mathrm{G}$ is the local magnetic field at LOFAR and $0.243 \mathrm{G}$ is the magnetic field strength at the Auger site. Details of Equation 2.4 and the parametrization of the charge-excess fraction are found in [2]. The radiation energy is increased by $11 \%$ to correct for the electron multiple scattering length factor in the underlying 
EGS4 simulation, following [15]. To compare to measured data, the radiation energy is scaled by $f_{r}^{2}$ from Equation 2.1.

We next find conversions between the radiation energy and the electromagnetic (energy deposited in the atmosphere by electromagnetic components of the shower) and total cosmic ray energies based on CoREAS simulations. Simulations for existing LOFAR analyses are used, consisting of 4900 proton and 4900 iron showers, ranging from $10^{16.5}-10^{18} \mathrm{eV}$, with zenith angles between $0^{\circ}-50^{\circ}$, and azimuth angles between $0^{\circ}-360^{\circ}$. We find the radiation energy for each as described above and relate them to the electromagnetic energy and primary cosmic ray energy by fitting the power law equation

$$
S_{R D}=A \times 10^{7} \mathrm{eV}\left(\frac{E}{10^{18} \mathrm{eV}}\right)^{B} .
$$

The results of this fit are shown in Figure 1. The radiation energy as a function of the electromagnetic energy is shown in the left panel, and as a function of total cosmic-ray energy in the right panel. The radiation energy is expected to scale quadratically with the energy contained in the electromagnetic components of the shower, and indeed that is seen. In the case of total energy, the difference between primary species is more evident. This is due to the fact that the total cosmicray energy includes the invisible energy from particles that do not release all their energy into the atmosphere, which is not measurable by radio antennas [16].
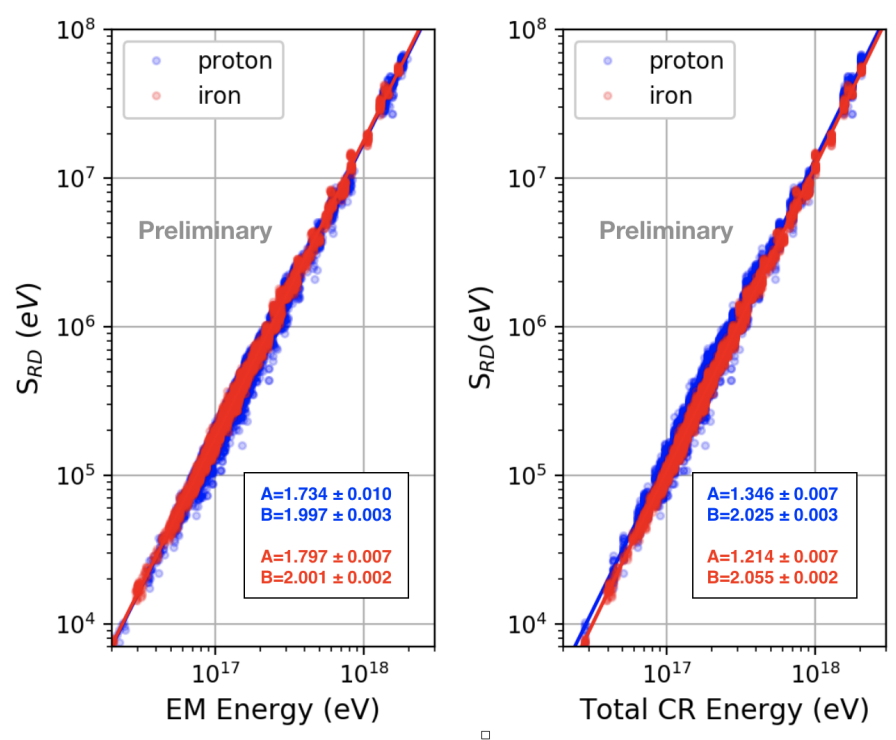

Figure 1: Radiation energy as a function of electromagnetic energy (left) and total (right) air shower energy. Proton events are shown in red, and iron in blue. Parameters A and B are best fit values for Equation 2.5, and are detailed in Table 1.

The values for fit parameters A and B found for electromagnetic energy are similar to those found in [2], and detailed in Table 1. Differences in the parameters may be due to the fact that a second order correction due to atmospheric density used in [2] is not included here. Also, the simulation sets in each case are not identical, including different energy ranges, zenith angles, and locations, although most differences are accounted for by the corrections made in Equation 2.4. 
Table 1: Best-fit parameters of Equation 2.5 for simulated proton and iron showers, which yield an energy estimation for the electromagnetic and total energy contained in the shower.

\begin{tabular}{||ccc||}
\hline & A & B \\
\hline \hline Proton EM energy & $1.734 \pm 0.010$ & $1.997 \pm 0.003$ \\
\hline Iron EM energy & $1.797 \pm 0.007$ & $2.001 \pm 0.002$ \\
\hline mixed EM energy from [2] & $1.629 \pm 0.003$ & $1.98 \pm 0.001$ \\
\hline Proton total energy & $1.346 \pm 0.007$ & $2.025 \pm 0.003$ \\
\hline Iron total energy & $1.214 \pm 0.007$ & $2.055 \pm 0.002$ \\
\hline
\end{tabular}

\section{LOFAR energy scale}

In this section we study the energy scale of LOFAR events based on radio and particle data and make a comparison of the two.

\subsection{Radio-based energy scale}

In order to calculate the total cosmic-ray energy on an event-to-event basis using Equation 2.5, the primary composition has to be taken into account, as there is a difference between proton and iron fit parameters for total energy fits. We use total energy, and not electromagnetic energy, because we will compare the radio-based energy measurements with the particle-based measurements which yield the total cosmic-ray energy. For each event, we choose the composition of the best fit simulation, as described in Section 2 and [5]. We first check that fluence-based method of determining energy is consistent with the direct scaling method. This is shown in Figure 2. The left panel shows a scatter plot of the cosmic-ray energy for each LOFAR event found using the direct scaling method $\left(E_{\text {dir.scal. }}\right)$ vs the fluence-based method $\left(E_{\text {flu. }}\right)$. The right panel shows the relative differences between the two methods, where there is no offset.
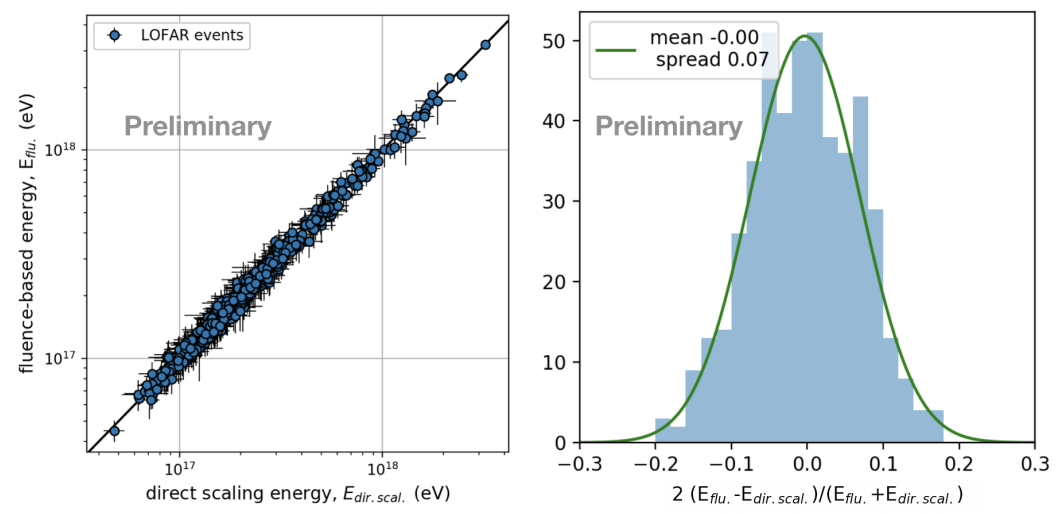

Figure 2: Left: Comparison of the energy found using the direct scaling method and the fluence-based method. Error bars indicate systematic uncertainties. Right: Relative differences between methods. 


\subsubsection{Uncertainties on radio-based energy scale}

The antenna calibration is the largest contribution to the systematic uncertainties, at $13 \%$ [7]. Uncertainties in the fit procedure have been studied in [5], where it was shown that the method resulted in no systematic offsets in the reconstructed core position or energy. While the electromagnetic energy of the shower has little dependence on hadronic interaction models, some uncertainty is introduced when we use the total shower energy. From [17], we see that the differences in invisible (non-calorimetric) energy due to hadronic interaction models are less than $4 \%$ for energies above $10^{17} \mathrm{eV}$, which we take as a systematic uncertainty on choice of model. In general, the uncertainties introduced using radiation energy from particle-level simulations translate into $2.6 \%$ in cosmic-ray energy $[2,15]$. The systematic uncertainties sum in quadrature to $14 \%$.

The statistical uncertainties are derived from a Monte Carlo study. For each event, a set of simulations is produced with the same energy and geometry. One simulation is taken to be truth, remaining simulations are fitted to it in order to reconstruct the energy and core position. This method is repeated for all the showers in the ensemble. Each reconstruction results in a scale factor $f_{r}$ from Equation 2.1. The standard deviation of the set of scale factors $f_{r}$ sets the statistical uncertainty of the event energy.

\subsection{Particle-based energy scale}

We compare the energy scale now established with the fluence-based method with the energy derived from particle measurements. The particle energy scale is also derived using a simulationbased approach. The best fitting CORSIKA simulation as determined by the $X_{\max }$ analysis is used. The particle distribution on the ground is binned into distance from shower core, and a GEANT4 [18] simulation of the LORA detectors converts particles that would produce a signal to energy deposited in the scintillators $[5,19]$. One challenge with the LORA particle data is that shower cores outside the superterp are difficult to constrain based on particle data alone. For this reason, the radio-based core location is used. Then, a particle $\chi^{2}$ is fit, as

$$
\chi^{2}=\sum_{\substack{\text { particle } \\ \text { detectors }}}\left(\frac{\mathrm{d}_{\mathrm{det}}-f_{p} \mathrm{~d}_{\mathrm{sim}}}{\sigma_{\mathrm{det}}}\right)^{2}
$$

where, $\mathrm{d}_{\mathrm{det}}$ is the deposited energy measured by a LORA detector with noise $\sigma_{\text {det }}$, and $\mathrm{d}_{\text {sim }}$ is the GEANT4 simulated deposit. The particle energy, $E_{\text {part. }}$, is then the simulated energy multiplied by the particle scale factor $f_{p}$.

\subsubsection{Uncertainties on particle-based energy scale}

Systematic uncertainties on the particle-based energy scale are estimated following [19]. Uncertainties in the deposited energy are on the order of $30 \%$. There are also uncertainties in the losses in the signal chain (cable losses, reflections) that contribute $15 \%$. These two contributions yield a systematic uncertainty of $33 \%$ on energy. Statistical uncertainties are handled in the same manner as for radio data. The set of simulations produced for the fitting procedure is used to reconstruct a mock data set, resulting in a set of $f_{p}$ scale factors, the standard deviation of which is used to set the statistical uncertainty for the event energy. 


\subsection{Comparison of radio and particle energy scales}

The comparison between radio-based energy and particle-based energy is shown in Figure 3. In the left panel, the LOFAR radiation energy $\left(S_{\mathrm{RD}}\right)$ is shown as a function of the cosmic-ray energy derived from the LORA scintillators $\left(E_{\text {part. }}\right)$. The error bars indicate statistical uncertainties. The predictions for radiation energy based on the total cosmic-ray energy from the fit parameters for proton and iron showers (from Equation 2.5) are shown as blue and red dashed lines. The radiation energy prediction from the AERA experiment is shown in green [3]. The right panel shows the relative differences in total cosmic-ray energy reconstructed with radio fluence $\left(E_{\text {flu. }}\right)$ and particle $\left(E_{\text {part. }}\right)$ methods.
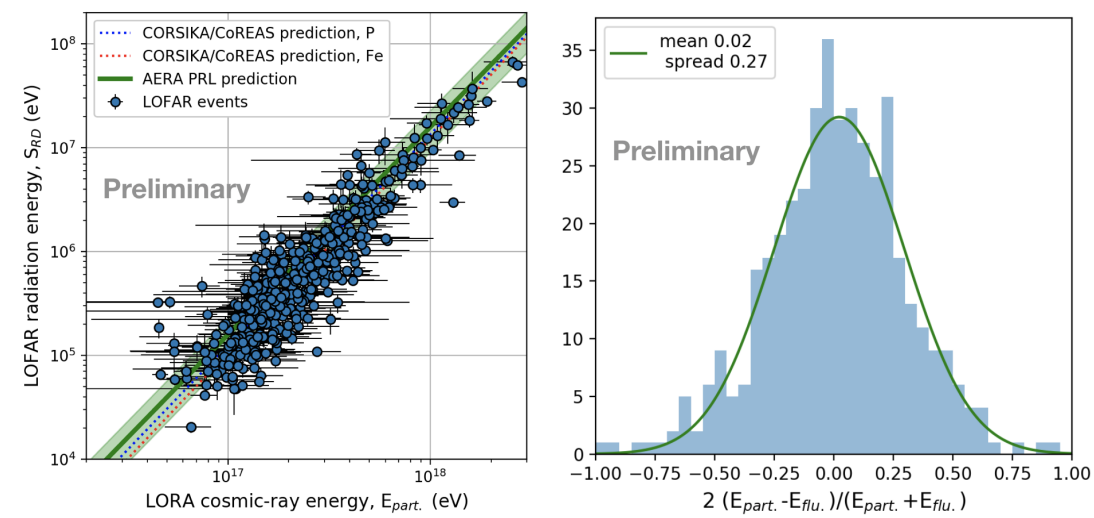

Figure 3: Left: Radiation energy as a function of cosmic-ray energy determined by LORA measurements. Also shown are predictions from CORSIKA/CoREAS with fit parameters described above, and expectations based on AERA results. Right: Relative difference between cosmic-ray energy determined with radio and particle data.

\section{Summary}

It has been shown that energy reconstruction using the radiation energy provides a universal, calorimetric energy measurement $[2,3]$. We have now established that the LOFAR energy scale measured this way is consistent with previous radio-based measurements, and also with the particle-based LORA energy scale. Understanding the energy scale has important consequences for the interpretation of other air shower properties measured with LOFAR, such as $X_{\max }$. Going forward, this technique will also allow measurements from different experiments to be directly compared.

\section{Acknowledgements}

The LOFAR cosmic-ray key science project acknowledges funding from an Advanced Grant of the European Research Council (FP/2007-2013) / ERC Grant Agreement n. 227610. The project has also received funding from the European Research Council (ERC) under the European Union's Horizon 2020 research and innovation programme (grant agreement No 640130). We furthermore acknowledge financial support from FOM, (FOM-project 12PR304). TW is supported by DFG grant WI 4946/1-1. AN is supported by the DFG grant NE 2031/2-1. LOFAR, the Low Frequency 
Array designed and constructed by ASTRON, has facilities in several countries, that are owned by various parties (each with their own funding sources), and that are collectively operated by the International LOFAR Telescope foundation under a joint scientific policy.

\section{References}

[1] T. Huege. Radio detection of cosmic ray air showers in the digital era. Physics Reports, 620:1-52, 2016.

[2] C. Glaser, M. Erdmann, J. R. Hörandel, T. Huege, and J. Schulz. Simulation of Radiation Energy Release in Air Showers. JCAP, 1609(09):024, 2016.

[3] A. Aab et al. Energy Estimation of Cosmic Rays with the Engineering Radio Array of the Pierre Auger Observatory. Phys. Rev., D93(12):122005, 2016.

[4] M. P. van Haarlem et al. LOFAR: The LOw-Frequency ARray. Astronomy and Astrophysics, 556:56, 2013.

[5] S. Buitink et al. Method for high precision reconstruction of air shower $\mathrm{X}_{\max }$ using two-dimensional radio intensity profiles. Phys. Rev. D, 90(8), 2014.

[6] P. Schellart et al. Detecting cosmic rays with the LOFAR radio telescope. Astronomy and Astrophysics, 560(A98), 2013.

[7] K. Mulrey et al. Calibration of the LOFAR low-band antennas using the Galaxy and a model of the signal chain. Astropart. Phys., 111:1-11, 2019.

[8] A. Corstanje et al. Xmax reconstruction and mass composition of cosmic rays with LOFAR. PoS, ICRC2019, 2019.

[9] D. Heck et al. CORSIKA: A Monte Carlo code to simulate extensive air showers. Report FZKA, 6019, 1998.

[10] T. Huege, M. Ludwig, and C. W. James. Simulating radio emission from air showers with CoREAS. AIP Conf. Proc., 1535:128, 2013.

[11] A. Ferrari et al. FLUKA: a multi-particle transport code. CERN-2005-10, INFN/TC_05/11, 2005.

[12] S. Ostapchenko. Monte Carlo treatment of hadronic interactions in enhanced Pomeron scheme: QGSJET-II model. Phys. Rev. D, 83(014018), 2011.

[13] A. Nelles et al. A parameterization for the radio emission of air showers as predicted by CoREAS simulations and applied to LOFAR measurements. Astropart.Phys., 60:13-24, 2015.

[14] P. Mitra et al. Reconstructing air showers with LOFAR using event specific GDAS atmospheres. PoS, ICRC2019, 2019.

[15] M. Gottowik, C. Glaser, T. Huege, and J. Rautenberg. Determination of the absolute energy scale of extensive air showers via radio emission: systematic uncertainty of underlying first-principle calculations. Astropart. Phys., 103:87-93, 2018.

[16] J. Linsley. Proc. of the 18th International Cosmic Ray Conference, Bangalore, India, 12:144, 1983.

[17] A. Aab et al. Data-driven estimation of the invisible energy of cosmic ray showers with the Pierre Auger Observatory. Submitted to: Phys. Rev. D, 2019.

[18] S. Agostinelli et al. GEANT4 -a simulation toolkit. NIMPA, 506(3):250-303, 2003.

[19] S. Thoudam et al. LORA: A scintillator array for LOFAR to measure extensive air showers. Nucl.Instrum.Meth, A767:339-346, 2014. 